\title{
Transcranial Direct-Current Stimulation (tDCS) Versus Venlafaxine ER In The Treatment Of Depression: A Randomized, Double-Blind, Single-Center Study With Open-Label, Follow-Up
}

This article was published in the following Dove Press journal:

Neuropsychiatric Disease and Treatment

\author{
Martin Bares $\mathbb{D}^{1,2}$ \\ Martin Brunovsky $\mathbb{D}^{1,2}$ \\ Pavla Stopkova (iD) 1,2 \\ Martin Hejzlar (iD) ${ }^{1,2}$ \\ Tomas Novak (D) ${ }^{1,2}$ \\ 'NIMH Clinical Center, National Institute \\ of Mental Health Czech Republic, \\ Topolova 748, Klecany, Czech Republic; \\ ${ }^{2}$ The Department of Psychiatry and \\ Medical Psychology, 3rd Faculty of \\ Medicine, Charles University, Prague, \\ Czech Republic
}

Objective: Transcranial direct-current stimulation (tDCS), a relatively new neuromodulation approach, provides some evidence of an antidepressant effect. This randomized, 4-week, doubleblind study with 8-week, open-label, follow-up compared the efficacy and tolerability of left anodal tDCS with venlafaxine ER (VNF) in the treatment of depression and prevention of early relapse. Methods: Subjects $(\mathrm{n}=57)$ received tDCS $(2 \mathrm{~mA}, 20$ sessions, 30 mins $)$ plus placebo $(\mathrm{n}=29)$ or VNF plus sham tDCS $(n=28)$. Responders to both interventions entered the open-label follow-up. The primary outcome was score change in the Montgomery-Åsberg Depression Rating Scale (MADRS) at week 4 of the study. Secondary outcomes were response, remission, dropout rates and relapse rates within the follow-up.

The mean change in the MADRS score from baseline to week for patients treated with tDCS was 7.69 (95\% CI, 5.09-10.29) points and 9.64 (95\% CI, 6.20-13.09) points for patients from the VNF group, a nonsignificant difference $(1.95,95 \%$ CI $-2.25-6.16 ; t(55)=0.93$, $\mathrm{p}=0.36$, Cohen's $d=0.24)$. There were no significant between-group differences in the MADRS scores from baseline to endpoint (intention-to-treat analysis). The response/remission rate for tDCS $(24 \% / 17 \%)$ and VNF $(43 \% / 32 \%)$ as well as the dropout rate (tDCS/VNF; $6 / 6)$ did not differ significantly between groups. In the follow-up, relapse (tDCS/VNF; 1/2) and dropout (tDCS/VNF; 2/3) rates were low and comparable.

Limitations: A relatively small sample size and short duration of the antidepressant treatment; no placebo arm.

Conclusion: Overall, this study found a similar efficacy of tDCS and VNF in the acute treatment of depression and prevention of early relapse. The real clinical usefulness of tDCS and its optimal parameters in the treatment of depression should be further validated.

Keywords: transcranial direct-current stimulation, tDCS, depression, venlafaxine ER, treatment

\section{Background}

Major depressive disorder (MDD) is a common, prevalent, recurrent and frequent chronic disorder associated with a high risk of mortality, morbidity and disease burden. ${ }^{1,2}$ Despite adequate treatment, a large proportion of patients $(>30 \%)$ have not achieved a significant clinical benefit. ${ }^{3}$ Noninvasive brain stimulation techniques (repetitive transcranial magnetic stimulation [rTMS], theta-burst stimulation and transcranial directcurrent stimulation [tDCS] ) emerged as alternative strategies based on knowledge about
Correspondence: Martin Bares

National Institute of Mental Health, Topolova 748, Klecany, Czech Republic

Tel +4 20266003330

Fax +4 20283088420

Email martin.bares@nudz.cz 
specific brain areas engaged in the pathophysiology of MDD. tDCS involves the application of a low-amplitude electrical direct current (1-2.5 mA) through two surface scalp electrodes (anode and cathode) to superficial areas of the brain. ${ }^{4}$ The putative therapeutic effect of tDCS in MDD is hypothesized to be based on the modulation of dysregulated cortical activity (hypoactivity of the left dorsolateral prefrontal cortex (DLPC), hyperactivity of right DLPC), connectivity and excitability that has been linked with amelioration of negatively biased information processing. ${ }^{5}$ The area under the anode is depolarized and the region under the cathode is hyperpolarized, leading to polarity-dependent changes on cortical excitability. ${ }^{6}$ Compared to rTMS, TDCS provides less focal stimulation of larger regions between the anode and the cathode; tDCS with the anode positioned over the left DLPC (placed on the F3 electrode in 10-20 International System) leads to the modulation of large-scale resting-state network connectivity. 5,7

The antidepressant efficacy of tDCS has been assessed in various meta-analyses since 2011. More recent metaanalyses detected the superiority of active tDCS compared to the sham stimulation. ${ }^{8-10}$

Several studies have focused on evaluating the efficacy of active and sham tDCS, whereas only a few trials have addressed the efficacy of tDCS compared to antidepressants (ADs); however, all of the studies used a selective serotonin reuptake inhibitor (SSRI; fluoxetine, sertraline, escitalopram) as a comparator. ${ }^{11-13}$

The antidepressive effect of both fluoxetine and sertraline was comparable to the antidepressive effect of tDCS, while escitalopram was associated with a better outcome. ${ }^{11-13}$

To date, there are only few open-label, uncontrolled studies examining the efficacy of tDCS for the continuation treatment of MDD. ${ }^{14,15}$

This double-blind, randomized study with open-label follow-up was designed to compare the efficacy and tolerability of tDCS and venlafaxine ER (VNF) in the acute treatment and prevention of early relapse. The primary outcome was changes in the conventional rating scale at the end of the double-blind phase. We also investigated the effects of tDCS versus VNF over time, assessed tolerability of both interventions and evaluated the relapse rate in the follow-up.

\section{Materials And Methods Study Design}

The trial was conducted at the National Institute of Mental Health Czech Republic (NIMH-CZ) in Klecany, with a recruitment period from October 2015 through March
2019. The NIMH-CZ Institutional Review Board reviewed and approved the study, and a written informed consent to participate in the research was obtained from all subjects. This clinical trial (EudraCT number 2015-001639-19; European Clinical Trials Database of European Medicines Agency) was regulated by the State Institute for Drug Control of Czech Republic (SUKL), and the study was also registered in the International Standard Randomized Controlled Trials Number (ISRCTN; ISRCTN93220632) registry. The design adhered to the latest version of the Declaration of Helsinki and ICH/ Good Clinical Practice guidelines. This single-center study comprised two phases: the first was a 4-week, twoarm double-blind, randomized trial. Following an initial washout period (2-7 days), recruited subjects were randomly allocated, according to a permuted block design with a fixed block size 4 , in a 1:1 ratio (no stratification) to either tDCS + placebo group or VNF + sham tDCS. The second phase was an 8-week, open-label, follow-up in which responders to both interventions from the doubleblind study continued to receive VNF or tDCS (performed once a week).

\section{Subjects}

We recruited patients with the diagnosis of MDD (recurrent or single episode) without psychotic symptoms according to DSM IV TR criteria, confirmed using The Mini - International Neuropsychiatric Interview - M.I.N. I., Czech version 5.0.0. ${ }^{16,17}$ All patients were hospitalized in the open ward of NIMH-CZ during the double-blind period of the study. They were admitted by referral from a number of outpatient clinics and psychiatric hospitals in the Czech Republic due to an unsatisfactory response to previous treatment. The study was advertised on the website of NIMH-CZ. Subjects fulfilled at least Stage I criteria for resistant depression ( $\geq 1$ adequate antidepressant treatment in the current episode) according to Thase and Rush. ${ }^{18}$ The evaluation of the adequacy of the previous medication in the index episode was based on the Antidepressant Treatment History Form (ATHF) with a score of at least $3 .^{19}$

We included right-handed subjects, 18 to 64 years old, who reached a total score of at least 25 in the MontgomeryÅsberg Depression Rating Scale (MADRS) ${ }^{20}$ and $\geq 4$ points in the Clinical Global Impression (CGI). ${ }^{21}$ The exclusion criteria were psychiatric comorbidity on axis I (including anxiety disorders) and II (DSM IV TR), history of psychotic and bipolar disorders, pregnancy or breastfeeding, serious 
unstable medical illness, unsuccessful treatment with VNF or tDCS in the current episode, contraindications of tDCS (e.g., metal in cranium, skull defects and skin lesions on the scalp) and neurologic disorders (epilepsy, brain injury and risk of seizure). The standard physical examination, medical history evaluation, psychiatric examination and biochemistry were performed to exclude risks and somatic or psychiatric comorbidities. Finally, previous fluoxetine treatment before the enrollment was the last exclusion criterion due to its long biological half-life.

\section{Study Treatment TDCS Group (TDCS + Placebo Capsules)}

Stimulation was performed using an HDCkit (Newronika s.r.l., Italy distributed by Magstim) that is a modular tDCS kit, consisting of a stimulator (HDCstim), a programmer (HDCprog) and a set of electrodes (HDCel). The anode and the cathode electrodes $(5 \times 5 \mathrm{~cm})$ were placed over the left and right DLPC (corresponding to F3 and F4 areas according to International 10-20 electroencephalographic system). tDCS was administered for 30 mins per session at $2.0 \mathrm{~mA}$. The first 20 sessions (4-week, double-blind phase) were applied for 20 consecutive workdays (double-blind phase of the study), and the remaining 8 sessions (open-label, follow-up for responders), with unchanged parameters, were applied once a week until week 12. Placebo capsules were administered using the same schedule as VNF (see below).

\section{VNF Group (Venlafaxine ER + Placebo Stimulation)}

Patients assigned to the VNF group took a dose of at least $150 \mathrm{mg}$ per day of VNF from the second week of the study. The dose could be increased by $75 \mathrm{mg}$, every 5 days, to a maximal dose of $375 \mathrm{mg}$ per day according to the clinical judgment of the attending physician. The dose could be decreased to $150 \mathrm{mg}$ per day for safety and tolerability reasons. Patients who did not tolerate at least $150 \mathrm{mg}$ of VNF per day were excluded from the study. The drug was dispensed in capsules identical to the placebo. For sham (placebo) tDCS, the same protocol was used as that used for active stimulation, but the device was automatically turned off after $60 \mathrm{~s}$ to mimic the typical initial sensation of tDCS. ${ }^{22}$ Responders were maintained on the efficient dose of VNF in a non-blind manner for an additional 8 weeks of follow-up.

\section{Concomitant Treatment}

The only concomitant treatments allowed were hydroxyzine (up to $100 \mathrm{mg}$ per day) for anxiety and zolpidem (up to 10 $\mathrm{mg}$ ) for insomnia. The continuation of benzodiazepine was allowed in an unchanged dosage in patients who used this medication before the study.

\section{Clinical Assessment}

Assessments were performed by experienced clinical psychiatrists (M.B., T.N. and M.H.) before the washout period, at baseline, and at weekly intervals to week 4 and then at the 2-week interval up to week 12 (the end of openlabel, follow-up). Raters were trained in the criterion of intraclass correlation $>0.80$ for each clinician prior to conducting ratings. ${ }^{23}$

The depressive symptoms and overall clinical status were evaluated using MADRS, CGI, Beck Depression Inventory-Short Form (BDI-SF) ${ }^{24}$ and Quick Inventory of Depressive Symptoms-Self-Rated (QIDS-SR). ${ }^{25}$ To assess the safety and side effects of both interventions, we questioned patients about adverse events and applied the tDCS Adverse Effects Questionnaire proposed by Brunoni in both treatment groups in the double-blind study. $^{12,22}$ This questionnaire evaluates the intensity of the potential of side effects of tDCS and their relationship to the treatment. We reported all data from the questionnaire that were considered by the patients to be at least moderate intensive, regardless of group assignment.

At the completion of the double-blind phase of the study or at a time of premature discontinuation, patients were asked whether they thought they had received tDCS or VNF to assess the integrity of trial-group blinding.

The primary endpoint was a change in the MADRS score from baseline to week 4 . Secondary outcomes were response to treatment $(\geq 50 \%$ reduction of MADRS total score at week four), remission (MADRS total score $\leq 10$ points), the scores on the BDI-SF, CGI, QIDS-SR and dropout rates for any reason as well as relapse rates (MADRS total score $\geq 20$ points in combination with a score of 4 or more points in the CGI at the time of follow-up visits or a change of antidepressant treatment due to substantial worsening of clinical status) within the follow-up.

Efficacy analyses of the double-blind phase of the study were performed in the intention-to-treat (ITT) (all randomized patients who received at least one dose or stimulation of allocated treatment) and per-protocol (PP) (subset of patients who completed the treatment originally allocated) populations. Safety analyses were carried-out on the ITT dataset. 


\section{Statistical Analysis}

To compare responders and nonresponders in baseline demographic and clinical data, we used the unpaired $t$-test, Mann-Whitney $U$-test and Fisher's exact test, as appropriate.

The primary efficacy measure (change of total MADRS score at week 4) analysis was based on ITT data, with the last observation carried forward (LOCF) method using the unpaired $t$-test with Welch correction. The relative efficacy of both treatment groups was calculated as an effect size (Cohen's $d$ ) from the difference in reduction in rating scale scores (initial minus final mean score) divided by the pooled final standard deviation.

MADRS, BDI-SF and QIDS-SR scores in both groups were tested using a repeated measure of ANOVA with the Greenhouse-Geisser adjustment and Fisher's LSD post hoc tests. The frequency of responders $(\geq 50 \%$ reduction in MADRS score), remitters (MADRS score $\leq 10$ points), adverse effects, dropouts and relapses (in open-label phase of the study) in both groups were compared using Fisher's exact test.

In addition, the number needed to treat (NNT) to obtain one responder to VNF was calculated for VNF versus tDCS groups over the 4-week treatment (ITT, completers).

An exact significance level of 0.05 was adopted. The analyses were performed using SPSS version 23, MedCalc Software version 17.8.6 and Statistica version 9.1.

The sample size was based on the primary outcome in the double-blind phase of the study (change of MADRS total score over week 4). A power analysis indicated that a total sample size of 52 subjects would be sufficient to detect an effect size of 0.8 (unpaired $t$-test), with $81 \%$ power at a $5 \%$ level of statistical significance.

\section{Results}

\section{Patient Characteristics Double-Blind Phase}

Altogether, 323 subjects that were hospitalized at the $2^{\text {nd }}$ Inpatient Ward: Mood Disorders of NIMH-CZ, from October 2015 to March 2019, were assessed for eligibility and 57 were randomly assigned to the treatment with tDCS $(n=29)$ or VNF $(n=28)-$ see Figure 1 . The discontinuation rate was not statistically different between the treatment groups (tDCS 6/29 (21\%), VNF 6/28 (21\%), $p=1.0)$ and 45 patients (79\% ITT sample) completed the entire doubleblind protocol. The ITT groups were similar in baseline clinical and demographic characteristics - see Table 1.
The average number of previous unsuccessful antidepressant treatments of index episode was 1.5 (min-max range, $1-4)$ in the entire sample. The average final dose of VNF was $220 \mathrm{mg}$ per day (min-max range, 150-300 mg).

\section{Open-Label, Follow-Up Phase}

Nineteen patients (responders to both interventions, tDCS: $\mathrm{n}=7,24 \%$; VNF: $\mathrm{n}=12,43 \%$ ) entered the open-label phase of the study. Five of them dropped out (tDCS: $n=2$; VNF: $n=3$ ). Two subjects from the tDCS group dropped out; the first of them relapsed and the second one switched to hypomania. Two patients from the VNF group were excluded from the study due to relapse and the third one withdrew consent due to personal reasons. Fourteen subjects $(25 \%$ of randomized subjects) completed the entire trial (double-blind and open-label phases) - see Figure 1.

\section{Efficacy And Safety Measures \\ Double-Blind Phase}

Fifty-seven subjects ( 36 females and 21 males, mean age $45.6 \pm 12.3$ ) were included in the efficacy analyses (ITT) (VNF: $n=28$; tDCS: $n=29$ ). The mean change in the total MADRS score from baseline to week 4 for patients treated with tDCS was 7.69 (95\% CI, 5.09-10.29) points and 9.64 (95\% CI, 6.20-13.09) points for patients from the VNF group, with nonsignificant difference between groups $(1.95,95 \%$ CI $-2.25-6.16 ; t(55)=0.93, p=0.36$, Cohen's $d=0.24)$. There was no significant difference between treatment groups in the MADRS scores (time $\times$ group interaction, $\mathrm{F}(4,220)=2.23, p=0.07)$ during the study - see Figure 2 and Table 2. Post hoc tests revealed differences in the MADRS score comparing baseline in both groups from week 1 to the end of the study. The PP analyses revealed effect of time $(F(4,172)=47.15$, $p<0.001)$ and time $\times$ group interaction $(\mathrm{F}(4,172)=3.76$, $p=0.006)$, but the post hoc test did not prove intergroup differences in any visit.

We also did not find any between-group differences in the other rating scales (CGI: $\mathrm{F}(4,220)=1.43, p=0.23$; QIDS-SR: $\mathrm{F}(4,220)=0.99, p=0.41$; BDI-SF: $\mathrm{F}(4,220)=$ $1.11, p=0.35)$. Both treatment groups experienced a significant reduction in CGI, QIDS SR and BDI-SF scores (for numerical details see Table 2).

The clinical response and remission rates between tDCS and VNF groups were not different in the ITT or PP analyses. The numerical details and NNT for response to VNF compared to tDCS are displayed in Table 3. 


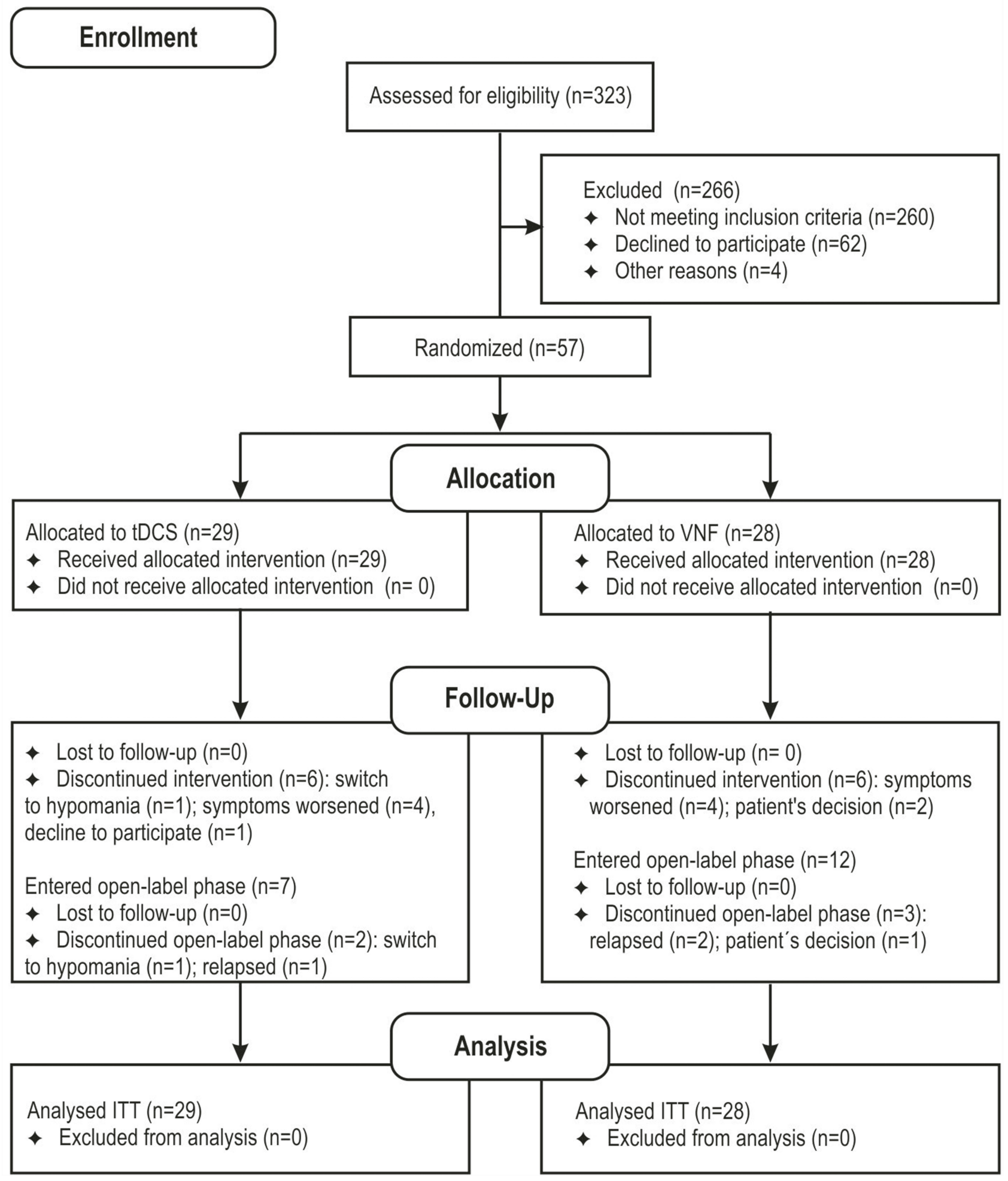

Figure I CONSORT flowchart.

Abbreviations: DB, double-blind; ITT, intention-to-treat; tDCS, transcranial direct-current stimulation; VNF; venlafaxine.

The final doses of venlafaxine (ITT) in responders (200 were not significantly different ( $\mathrm{t}(26)=1.47, p=0.17)$. $\pm 48.85 \mathrm{mg})$ and nonresponders $(234.38 .4 \pm 71.81 \mathrm{mg})$ The number of subjects who took benzodiazepines 
Table I Baseline Demographic And Clinical Characteristics Of Patients (Intention-To-Treat Sample)

\begin{tabular}{|c|c|c|c|}
\hline & $\operatorname{tDCS}(n=29)$ & VNF $(n=28)$ & p Value \\
\hline Age (years) & $46.6 \pm 13.0$ & $44.6 \pm 11.7$ & $0.41^{\mathrm{a}}$ \\
\hline Sex (F:M) & $17: 12$ & 19:9 & $0.59^{\mathrm{b}}$ \\
\hline Illness duration (month) & $82.9 \pm 90.9$ & $98.3 \pm 83.4$ & $0.5 I^{\mathrm{a}}$ \\
\hline Number of previous episodes & $1.6 \pm 1.8$ & $2.1 \pm 1.8$ & $0.47^{c}$ \\
\hline Duration of index episode before enrollment (weeks) & $29.5 \pm 23.8$ & $32.4 \pm 27.4$ & $0.68^{\mathrm{a}}$ \\
\hline Number of previous adequate treatment trials of index episode & I. \pm 0.7 & $1.6 \pm 0.8$ & $0.7^{c}$ \\
\hline Baseline MADRS score & $27.7 \pm 2.8$ & $28.3 \pm 3.3$ & $0.49^{\mathrm{a}}$ \\
\hline Baseline BDI-SF score & $18.6 \pm 6.4$ & $19.2 \pm 6.9$ & $0.72^{\mathrm{a}}$ \\
\hline Baseline CGI score & $4.3 \pm 0.4$ & $4.3 \pm 0.5$ & $0.57^{\mathrm{a}}$ \\
\hline Baseline QIDS-SR score & $17.1 \pm 8.0$ & $16.4 \pm 5.0$ & $0.67^{\mathrm{a}}$ \\
\hline $\begin{array}{l}\text { Last treatment before } \\
\text { the enrollment }\end{array}$ & $\begin{array}{l}\text { AD+AP2: } 5 \\
\text { CAD: I0 } \\
\text { NDRI: } 2 \\
\text { RIMA: I } \\
\text { SARI: I } \\
\text { SSRI: } 9 \\
\text { vortioxetine: I }\end{array}$ & $\begin{array}{l}\text { AD+AP2: } 5 \\
\text { CAD: } 9 \\
\text { NaSSA: I } \\
\text { RIMA: I } \\
\text { SARI: I } \\
\text { SSRI:II }\end{array}$ & NA \\
\hline Treatment resistance $^{d}$ & 11 & 11 & $1.00^{\mathrm{b}}$ \\
\hline Number of subjects taking benzodiazepines at baseline ${ }^{e}$ & 7 & 5 & $0.53^{\mathrm{b}}$ \\
\hline
\end{tabular}

Notes: Values are mean \pm SD or number of subjects. ${ }^{a}$ unpaired $t$-test, ${ }^{b}$ Fisher's exact test, ${ }^{C}$ Mann-Whitney $U$-test, ${ }^{d} \geq 2$ adequate antidepressant trials, ${ }^{e}$ unchanged dose during the study. Abbreviations: AD+AP2, combination of antidepressant and antipsychotic of the second generation; BDI-SF, Beck Depression Inventory-Short Form; CAD, combination of antidepressants; CGI, Clinical Global Impression; MADRS, Montgomery and Åsberg Depression Rating Scale; NA, not applicable; NaSSA, noradrenergic and specific serotonergic antidepressant; NDRI, norepinephrine and dopamine reuptake inhibitor; QIDS-SR, Quick Inventory of Depressive Symptomatology Self-Report; RIMA, reversible inhibitor of monoaminoxidase; SARI, serotonin antagonist and reuptake inhibitor; SSRI, serotonin reuptake inhibitors; tDCS, transcranial direct-current stimulation; VNF, venlafaxine.

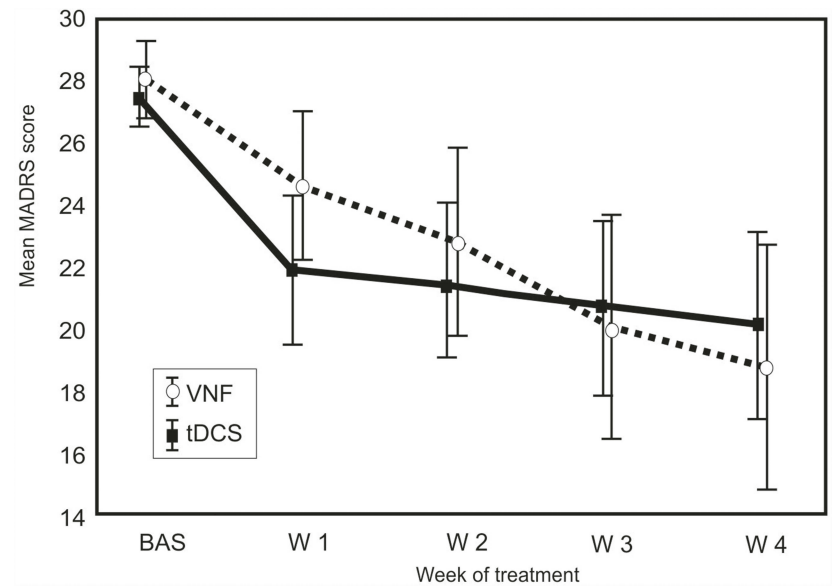

Figure 2 Change in mean MADRS scores between subjects treated with tDCS and VNF (intention-to-treat sample, $n=57$ ).

Notes: Values are mean \pm SD.

Abbreviations: BAS, baseline; MADRS, Montgomery and Åsberg Depression Rating Scale; tDCS, transcranial direct-current stimulation; VNF, venlafaxine; W, week. (unchanged dose in the study; see Table 1) or hydroxyzine and zolpidem (at the end of the study), in both groups, also did not differ.

Generally, both treatments were well tolerated. There was only one serious adverse event (switch to mania) that led to readmission into the psychiatric department (first week of tDCS treatment). Other reasons for treatment discontinuation were worsening of clinical status and the patient's decision to not continue in the study. The discontinuation rate was not different between groups (randomized sample: tDCS: $\mathrm{n}=6$ (21\%); VNF: $\mathrm{n}=6(21 \%), p=1.00)$ - see Figure 1.

The adverse event rate based on the tDCS Adverse Effects Questionnaire (item 1-10) and clinical interview is described in Table 4. The rate of individual adverse events did not differ between groups, except neck pain that was observed more frequently in the tDCS group (see 


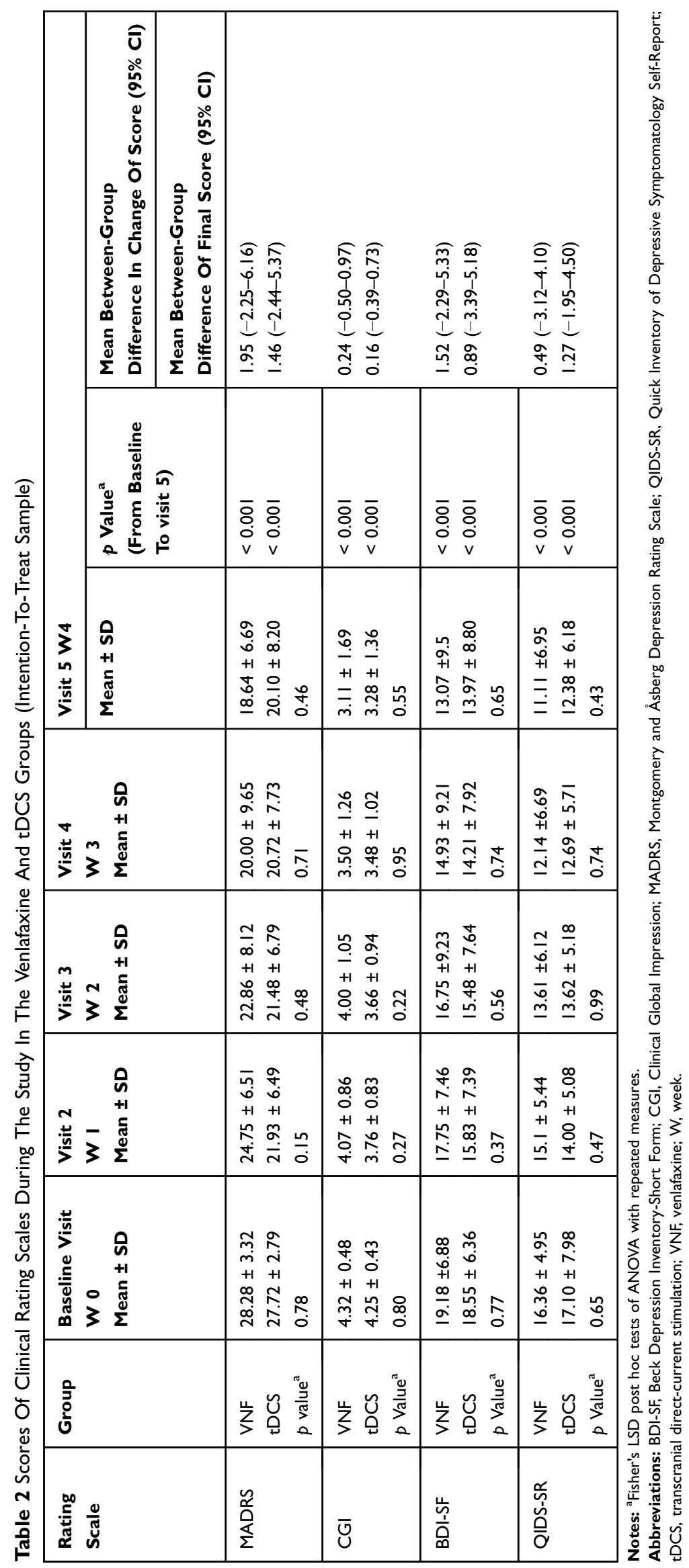


Table 3 Response And Remission Rate In tDCS And Venlafaxine Groups In The Double-Blind Phase Of The Study (Intention-To-Treat And Per Protocol Analyses)

\begin{tabular}{|l|l|l|l|l|l|}
\hline & Analysis & tDCS & VNF & $\boldsymbol{p ~ V a l u e ~}^{\mathbf{a}}$ & NNT $^{\mathbf{b}}$ \\
\hline Response rate (\%) & ITT & 24 & 43 & 0.17 & 5 \\
& PP & 30 & 55 & 0.14 & 4 \\
\hline Remission rate (\%) & ITT & 17 & 32 & 0.23 & 6 \\
& PP & 22 & 41 & 0.21 & 6 \\
\hline
\end{tabular}

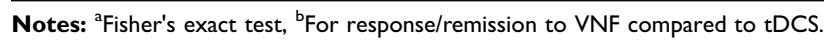

Abbreviations: ITT, intention-to-treat; NNT, number needed to treat; PP, per protocol; tDCS, transcranial direct-current stimulation; VNF, venlafaxine.

Table 4 Summary Of Adverse Events In Double-Blind Phase Of The Study

\begin{tabular}{|c|c|c|c|}
\hline Adverse Events & $\begin{array}{l}\text { tDCS } \\
(n=29)\end{array}$ & $\begin{array}{l}\text { VNF } \\
(n=28)\end{array}$ & $p$ Value $^{a}$ \\
\hline \multicolumn{4}{|l|}{ tDCS questionnaire ${ }^{b}$} \\
\hline Headache & $10(34 \%)$ & II (39\%) & 0.78 \\
\hline Neck pain & $7(24 \%)$ & I (4\%) & 0.06 \\
\hline Scalp pain & $5(17 \%)$ & $2(7 \%)$ & 0.42 \\
\hline Tingling & $3(10 \%)$ & $3(11 \%)$ & 1.00 \\
\hline Itching & 7 (24\%) & $3(11 \%)$ & 0.30 \\
\hline Burning sensations & $5(17 \%)$ & $3(11 \%)$ & 1.00 \\
\hline Skin redness & $9(3 \mid \%)$ & $5(18 \%)$ & 0.71 \\
\hline Sleepiness & $19(66 \%)$ & $17(61 \%)$ & 0.79 \\
\hline Trouble concentrating & $16(55 \%)$ & $16(27 \%)$ & 1.00 \\
\hline Acute mood change & $16(55 \%)$ & $13(46 \%)$ & 0.60 \\
\hline \multicolumn{4}{|l|}{ Clinical interview } \\
\hline Anxiety $^{c}$ & $15(52 \%)$ & $15(54 \%)$ & 1.00 \\
\hline Diarrhea & $0(0 \%)$ & I (4\%) & 0.49 \\
\hline High systolic blood pressure & $0(0 \%)$ & I (4\%) & 0.49 \\
\hline Hypomania (mania) & I (3\%) & $0(0 \%)$ & 1.00 \\
\hline Insomnia ${ }^{d}$ & $12(4 \mid \%)$ & $12(43 \%)$ & 1.00 \\
\hline Irritability & I (3\%) & $0(0 \%)$ & 1.00 \\
\hline $\begin{array}{l}\text { Skin lesion under stimulation } \\
\text { electrode }\end{array}$ & I (3\%) & $0(0 \%)$ & 1.00 \\
\hline Sweating & $0(0 \%)$ & I (4\%) & 0.49 \\
\hline
\end{tabular}

Notes: Values are number of events (\%). ${ }^{\mathrm{a}}$ Fisher's exact test, ${ }^{\mathrm{b}}$ adverse events with more than mild intensity, 'required concomitant treatment with hydroxyzine, ${ }^{d}$ required concomitant treatment with zolpidem.

Abbreviations: tDCS, transcranial direct-current stimulation; VNF, venlafaxine.

Table 4). The most frequent events in both groups were sleepiness, trouble concentrating, headache, acute mood change, anxiety and insomnia (the latter two items from clinical interviews).

\section{Follow-Up Phase Of The Study}

Nineteen responders (tDCS: $n=7, V N F: n=12$ ) entered the follow-up, open-label study. There was no difference between groups in the number of dropout patients (tDCS: $\mathrm{n}=2,29 \%$, VNF: $\mathrm{n}=3,25 \%, p=1.00)$ or relapses (tDCS: $\mathrm{n}=1 ;$ VNF: $\mathrm{n}=2, p=1.00)$.

\section{Integrity Of Blinding}

The proportions of patients in each group that guessed the type of treatment (tDCS 55\%, VNF 46\%) when they were asked at the end of the study or at the time of premature discontinuation were not significantly different $(p=0.60)$. The Fisher's exact test and unpaired $t$-test, which were used to assess differences between the response rates and changes in the MADRS score and guesses of treatment allocation, did not find significant differences between the groups (ITT sample, $p=1.0$ and 0.79 , respectively).

\section{Discussion}

Overall, in this single-center, randomized, double-blind study with an open-label follow-up that involved patients suffering from MDD, anodal tDCS over left DPLC did not differ from VNF in terms of efficacy, safety, tolerability or ability to prevent early relapse. The ITT and PP analyses provided the same pattern of results. Both treatment modalities achieved statistically significant changes (reduction) in depressive symptoms at the endpoint. Although statistical differences between the tDCS group and the VNF group in terms of response/remission rate were not demonstrated, the VNF group achieved a numerically higher proportion of positive outcomes. Both interventions were generally well tolerated and acceptable to patients. Except for the two patients who were excluded from the study due to treatment-related hypomania (tDCS group; one in the double-blind phase and a second one in the follow-up), no patient dropped due to side effects of the applied interventions. The treatment-emergent hypomania/mania during the tDCS treatment is considered a possible, infrequent side effect $(3.5 \%){ }^{22,26}$ According to our knowledge, the present study is the first one to compare 
tDCS in the treatment of MDD to another antidepressant other than SSRI. Moreover, the inpatient status of participants in this acute, double-blind phase of the project allowed precise control of the treatment regimen.

The different stimulation parameters (duration of sessions, the cathode placement and number of stimulations) make the comparison of our findings with other studies evaluating the efficacy and safety of ADs and tDCS difficult. $^{11-13}$

Rigonatti's study $(\mathrm{n}=42)$ compared tDCS $(2 \mathrm{~mA}$ of intensity for 20 mins for 10 days, anodal electrode on the left DLPC and cathode electrode on the contralateral supraorbital area) to sham tDCS and fluoxetine $(20 \mathrm{mg}$ per day). ${ }^{13}$ After 6 weeks, depression reduction (BDI) was similar in both of the active treatment groups. Interestingly, no boosted sessions were used after 2 weeks of treatment, and only a minor fluctuation of depressive symptoms was detected in the tDCS group between the second and sixth weeks of the study.

Brunoni's 6-week, double-blind study $(\mathrm{n}=120)$ assessed the efficacy of tDCS ( $2 \mathrm{~mA}$ of intensity for 30 mins, 12 applications (10 stimulations in 2 weeks +2 additional (boosted) sessions every other week, the anode positioned on left DLPC and the cathode on right DLPC) + placebo, sertraline (50 mg per day) + shame stimulation, placebo + sham stimulation, and sertraline + tDCS (SELECT TDCS). ${ }^{12}$ The combined treatment differed significantly from placebo, tDCS and sertraline. No difference was observed between tDCS and sertraline (mean difference 2.6 points) and sertraline did not reach statistical significance compared to placebo. Also, the response rate did not differ between sertraline and tDCS.

The recent double-blind, placebo-controlled study $(\mathrm{n}=245)$ comparing tDCS (2 mA, 15 sessions applied on workdays, subsequent 7 sessions took place weekly) + placebo to escitalopram (up to $20 \mathrm{mg}$ per day) + sham stimulation, and placebo + sham stimulation failed to show noninferiority of tDCS to escitalopram over a 10 -week period (ELECTTDCS). ${ }^{11}$ Escitalopram was superior to tDCS (mean difference of 2.3 points in Hamilton depressive rating scale ${ }^{27}$ ). However, the rates of response/remission to tDCS and escitalopram were not significantly different.

All three studies considered tDCS as a safe and welltolerated method.

Similar to the studies with fluoxetine and sertraline, ${ }^{12,13}$ we did not find differences in the reduction of depressive symptoms between tDCS and ADs at the end of the study. The mean difference of change in the MADRS score between treatment groups in our study was numerically lower than in Brunoni's studies (sertraline, escitalopram). ${ }^{11,12}$

None of the studies demonstrated differences between tDCS and ADs in the secondary outcome measures (response/remission rate). Scrutinizing their results, we found a lower response/remission rate of tDCS in our study compared to Brunoni's study (there was no mention of qualitative outcome in Rigonatti's study). VNF achieved results similar to sertraline and escitalopram.

As the position and optimal protocol for tDCS-treatment of MDD were not clearly established at the time of the designing the study, we used identical stimulation parameters (electrode size and placement and current strength) as SELECT-TDCS ${ }^{12}$ - with a higher overall number of stimulations that were in line with the recommendation that the European expert group has proposed for tDCS use in patients without treatment resistance $(>10$ sessions, 20-30 mins, the anode over left DLPC and the cathode over right supraorbital region). ${ }^{28}$ There are only limited data on continuation treatment with tDCS or ADs after the series of stimulations. ${ }^{14,15}$ There is no study that compares the efficacy of AD and tDCS in the continuation or maintenance treatment of MDD after successful tDCS intervention in the acute phase of MDD.

There are several caveats to our findings. First, the 4-week treatment period might be too short to assess the clinical response to VNF, as well as tDCS. We cannot exclude the possibility of a further clinical response to VNF emerging during longer treatment. However, a 4-week period is repeatedly used as the cutoff for an adequate antidepressant trial (ATHF). ${ }^{29}$ The recent treatment guidelines for MDD proposed a 4-week period as a point of decision on treatment changes. ${ }^{30,31}$ Results from older trials suggest that VNF may have an early onset of action and demonstrate substantial improvement in depressive symptomatology after 4 weeks of treatment. ${ }^{32-34}$ It is assumed that the longer and more intensive tDCS trial (number of stimulations, current intensity, etc.) would lead to a better antidepressant effect. ${ }^{5,35}$ The most recent projects have been designed for a 6- or 10-week active treatment period (including a 2- or 7-week extension, with tDCS applied once a week). ${ }^{5,36,37}$

Nevertheless, 22 stimulations applied in the ELECTTDCS study achieved a similar response rate or reduction of depressive symptoms as 12 stimulations in a previous study (SELECT-TDCS). ${ }^{11,12}$

The second limitation of this study is a relatively small sample size that was calculated for continuous variables 
and clinically meaningful interest. However, we only found a small-sized between-group difference in the MADRS score change (Cohen's $d=0.24$ ) at the end of the study and only approaching the minimal clinically important difference of MADRS (2 points). ${ }^{38}$ There was a high numerical difference between tDCS and VNF in the response/remission rate, and the NNT for response to VNF compared to tDCS achieved a range of clinical significance; therefore, we cannot exclude type II error. The analyses of categorical outcomes might have been underpowered.

A third possible limit is the lack of the placebo control arm, as both treatments have been previously reported to be more effective than placebo, ${ }^{4,8,9}$ and the Institutional Review Board of NIMH-CZ would not have approved a placebo-controlled project in the treatment of resistant patients.

Fourth, due to the double-blind assignment of the study, we applied a tool for assessment of adverse events that is more appropriate for tDCS. It only partially covered the side effects of $\mathrm{AD}$.

Finally, the inpatient status allowed us to control the treatment regimen, decrease the potential risk of selfinjury and facilitate the smooth running of the doubleblind phase of the study but might influence the treatment outcome because of higher expectation bias. Hence, the generalizability of our findings to the outpatient population should be interpreted with caution.

The most important and lasting task for future research will be to get unequivocal data and evidence for the acute and longterm efficacy of tDCS in larger studies. ${ }^{35}$ Above all, it is necessary to compare different types of treatment protocols in acute and continuation treatments (e.g., number of sessions, electrode size, influence of antidepressant co-medication etc.), to evaluate the efficacy of tDCS in the augmentation of various ADs, as well as to find a way to optimize treatment outcomes in resistant patients. The promising step might be the application of the high-definition tDCS (HD-tDCS), using an array of small electrodes (as small as those for electroencephalogram), which has been investigated in the context of better spatial resolution, increasing the focality. ${ }^{39}$

Similar to other antidepressant methods, there is also a clear clinical need to identify subjects that could have maximal benefit from tDCS - find predictors of treatment outcome. ${ }^{40}$ We hope that subsequent analyses of the study results focused on biomarkers of response (QEEG, rsfMRI) might be a part of this effort.
Based on our results, with the foregoing limitations, we presume that antidepressant treatments would be preferred over tDCS.

\section{Conclusion}

Overall, this study found similar efficacy and tolerability of both treatment modalities. The real clinical usefulness of tDCS monotherapy and its optimal parameters in the treatment of depression should be further validated.

\section{Data Sharing Statement}

The clinical and demographic data used to support the findings of this study are available from the corresponding author upon request.

\section{Acknowledgment}

Natalie Görnerova, Jiri Renka and Libuse Pohlova provided and cared for study patients.

\section{Author Contributions}

All authors (Bares, Brunovsky, Stopkova, Hejzlar, Novak) contributed to conception and design, participated in the clinical part of the project (treatment, ratings, etc.) and took part in analyses and interpretation of acquired data. All members of the team drafted the article, revised it, and approved the final version. All authors agreed to be accountable for all aspects of the work in ensuring that questions related to the accuracy or integrity of the work are appropriately investigated and resolved.

\section{Funding}

This study is supported by the Ministry of Health of the Czech Republic, grant AZV ČR (Czech Health Research Council) nr. 15-29900A.

\section{Disclosure}

Dr Martin Bareš and Dr Martin Brunovsky report grant from AZV ČR (Czech Health Research Council), during the conduct of the study (nr. 1529900A) and grants from AZV ČR nr.16-31380A and nr.15-33250A, ouside the submitted work. Dr Pavla Stopkova reports grant, personal fees from AZV ČR grant nr. 15-29900A, during the conduct of the study. She also received grant, personal fees from grant AZV MZ ČR nr. 16-29857A, outside the submitted work. Dr Martin Hejzlar reports grant from AZV $\breve{C} \mathrm{R}$ nr. 15-29900A, during the conduct of the study. Dr Tomas Novak reports grant from AZV ČR nr. 1529900A, during the conduct of the study; personal fees from Krka 
CR and grant from AZV ČR 16-31380A, outside the submitted work. The authors report no other conflicts of interest in this work.

\section{References}

1. Kessler RC, Bromet EJ. The epidemiology of depression across cultures. Annu Rev Public Health. 2013;34:119-138. doi:10.1146 annurev-publhealth-031912-114409

2. Olesen J, Gustavsson A, Svensson M, et al. The economic cost of brain disorders in Europe. Eur $J$ Neurol. 2012;19(1):155-162. doi:10.1111/j.1468-1331.2011.03590.x

3. Rush AJ, Trivedi MH, Wisniewski SR, et al. Acute and longer-term outcomes in depressed outpatients requiring one or several treatmen steps: a STAR*D report. Am J Psychiatry. 2006;163(11):1905-1917. doi:10.1176/ajp.2006.163.11.1905

4. Mutz J, Edgcumbe DR, Brunoni AR, Fu CHY. Efficacy and acceptability of non-invasive brain stimulation for the treatment of adult unipolar and bipolar depression: a systematic review and meta-analysis of randomised sham-controlled trials. Neurosci Biobehav Rev. 2018;92:291-303. doi:10.1016/j.neubiorev.2018.05.015

5. Padberg F, Kumpf U, Mansmann U, et al. Prefrontal transcranial direct current stimulation (tDCS) as treatment for major depression: study design and methodology of a multicenter triple blind randomized placebo controlled trial (DepressionDC). Eur Arch Psychiatry Clin Neurosci. 2017;267(8):751-766. doi:10.1007/s00406-017-0769-y

6. Borrione L, Moffa AH, Martin D, Loo CK, Brunoni AR. Transcranial direct current stimulation in the acute depressive episode: a systematic review of current knowledge. J Ect. 2018;34(3):153-163. doi:10.1097/YCT.0000000000000512

7. Pathak Y, Kopell BH, Szabo A, Rainey C, Harsch H, Butson CR. The role of electrode location and stimulation polarity in patient response to cortical stimulation for major depressive disorder. Brain Stimul. 2013;6(3):254-260. doi:10.1016/j.brs.2012.07.001

8. Brunoni AR, Moffa AH, Fregni F, et al. Transcranial direct current stimulation for acute major depressive episodes: meta-analysis of individual patient data. $B r \quad J$ Psychiatry. 2016;208(6):522-531. doi:10.1192/bjp.bp.115.164715

9. Meron D, Hedger N, Garner M, Baldwin DS. Transcranial direct current stimulation (tDCS) in the treatment of depression: systematic review and meta-analysis of efficacy and tolerability. Neurosci Biobehav Rev. 2015;57:46-62. doi:10.1016/j.neubiorev.2015.07.012

10. Shiozawa P, Fregni F, Bensenor IM, et al. Transcranial direct current stimulation for major depression: an updated systematic review and meta-analysis. Int J Neuropsychopharmacol. 2014;17(9):1443-1452. doi: $10.1017 / \mathrm{S} 1461145714000418$

11. Brunoni AR, Moffa AH, Sampaio-Junior B, et al. Trial of electrical direct-current therapy versus escitalopram for depression. $N$ Engl $J$ Med. 2017;376(26):2523-2533. doi:10.1056/NEJMoa1612999

12. Brunoni AR, Valiengo L, Baccaro A, et al. The sertraline vs. electrical current therapy for treating depression clinical study: results from a factorial, randomized, controlled trial. JAMA Psychiatry. 2013;70(4):383-391. doi:10.1001/2013.jamapsychiatry.32

13. Rigonatti SP, Boggio PS, Myczkowski ML, et al. Transcranial direct stimulation and fluoxetine for the treatment of depression. Eur Psychiatry. 2008;23(1):74-76. doi:10.1016/j.eurpsy.2007.09.006

14. Martin DM, Alonzo A, Ho KA, et al. Continuation transcranial direct current stimulation for the prevention of relapse in major depression. J Affect Disord. 2013;144(3):274-278. doi:10.1016/j.jad.2012.10.012

15. Valiengo L, Bensenor IM, Goulart AC, et al. The sertraline versus electrical current therapy for treating depression clinical study (select-TDCS): results of the crossover and follow-up phases. Depress Anxiety. 2013;30(7):646-653. doi:10.1002/da.22079

16. APA. Diagnostic and Statistical Manual of Mental Disorders. 4th. Washington DC: American Psychiatric Press; 2004.
17. Sheehan DV, Lecrubier Y, Sheehan KH, et al. The Mini-International Neuropsychiatric Interview (M.I.N.I.): the development and validation of a structured diagnostic psychiatric interview for DSM-IV and ICD-10. J Clin Psychiatry. 1998;59(Suppl 20):22-33.

18. Thase ME, Rush AJ. When at first you don't succeed: sequential strategies for antidepressant nonresponders. $J$ Clin Psychiatry. 1997;58(Suppl 13):23-29.

19. Sackeim HA. The definition and meaning of treatment-resistant depression. J Clin Psychiatry. 2001;62(Suppl 16):10-17.

20. Montgomery SA, Asberg M. A new depression scale designed to be sensitive to change. Br J Psychiatry. 1979;134:382-389. doi:10.1192/ bjp.134.4.382

21. Guy W. ECDEU Assessment Manual for Psychopharmacology Revised (DHEW Publ No ADM 76-338). Rockville, MD: US Department of Health, Education, and Welfare; Public Health Service; Alcohol, Drug Abuse, and Mental Health Administration; NIMH Psychopharmacology Research Branch; Division of Extramural Research Programs; 1976:218-222.

22. Brunoni AR, Amadera J, Berbel B, Volz MS, Rizzerio BG, Fregni F. A systematic review on reporting and assessment of adverse effects associated with transcranial direct current stimulation. Int $J$ Neuropsychopharmacol. 2011;14(8):1133-1145. doi:10.1017/S1461 145710001690

23. Kobak KA, Greist JH, Jefferson JW, Katzelnick DJ. Computer-administered clinical rating scales. A review. Psychopharmacology (Berl). 1996;127(4):291-301. doi:10.1007/s002130050089

24. Beck AT, Rial WY, Rickels K. Short form of depression inventory: cross-validation. Psychol Rep. 1974;34(3):1184-1186.

25. Rush AJ, Trivedi MH, Ibrahim HM, et al. The 16-Item Quick Inventory of Depressive Symptomatology (QIDS), clinician rating (QIDS-C), and self-report (QIDS-SR): a psychometric evaluation in patients with chronic major depression. Biol Psychiatry. 2003;54 (5):573-583. doi:10.1016/s0006-3223(02)01866-8

26. Matsumoto H, Ugawa Y. Adverse events of tDCS and tACS: a review. Clin Neurophysiol Pract. 2017;2:19-25. doi:10.1016/j. cnp.2016.12.003

27. Hamilton M. A rating scale for depression. J Neurol Neurosurg Psychiatry. 1960;23:56-62. doi:10.1136/jnnp.23.1.56

28. Lefaucheur JP, Antal A, Ayache SS, et al. Evidence-based guidelines on the therapeutic use of transcranial direct current stimulation (tDCS). Clin Neurophysiol. 2017;128(1):56-92. doi:10.1016/j.clinph.2016.10.087

29. Souery D, Oswald P, Massat I, et al. Clinical factors associated with treatment resistance in major depressive disorder: results from a European multicenter study. J Clin Psychiatry. 2007;68(7):10621070. doi:10.4088/jcp.v68n0713

30. Bauer M, Severus E, Moller HJ, Young AH, Disorders W. Pharmacological treatment of unipolar depressive disorders: summary of WFSBP guidelines. Int J Psychiatry Clin Pract. 2017;21 (3):166-176. doi:10.1080/13651501.2017.1306082

31. Kennedy SH, Lam RW, McIntyre RS, et al. Canadian Network for Mood and Anxiety Treatments (CANMAT) 2016 clinical guidelines for the management of adults with major depressive disorder: section 3. Pharmacological treatments. Can J Psychiatry. 2016;61(9):540560. doi: $10.1177 / 0706743716659417$

32. Benkert O, Grunder G, Wetzel H, Hackett D. A randomized, doubleblind comparison of a rapidly escalating dose of venlafaxine and imipramine in inpatients with major depression and melancholia. $J$ Psychiatr Res. 1996;30(6):441-451. doi:10.1016/s0022-3956(96) 00029-5

33. Debonnel G, Saint-Andre E, Hebert C, de MC, Lavoie N, Blier P. Differential physiological effects of a low dose and high doses of venlafaxine in major depression. Int $J$ Neuropsychopharmacol. 2007;10(1):51-61. doi:10.1017/S1461145705006413

34. Guelfi JD, White C, Hackett D, Guichoux JY, Magni G. Effectiveness of venlafaxine in patients hospitalized for major depression and melancholia. J Clin Psychiatry. 1995;56(10):450-458. 
35. Palm U, Hasan A, Strube W, Padberg F. tDCS for the treatment of depression: a comprehensive review. Eur Arch Psychiatry Clin Neurosci. 2016;266(8):681-694. doi:10.1007/s00406-016-0674-9

36. Alonzo A, Aaronson S, Bikson M, et al. Study design and methodology for a multicentre, randomised controlled trial of transcranial direct current stimulation as a treatment for unipolar and bipolar depression. Contemp Clin Trials. 2016;51:65-71. doi:10.1016/j. cct.2016.10.002

37. Brunoni AR, Sampaio-Junior B, Moffa AH, et al. The Escitalopram versus Electric Current Therapy for Treating Depression Clinical Study (ELECT-TDCS): rationale and study design of a non-inferiority, triple-arm, placebo-controlled clinical trial. Sao Paulo Med J. 2015;133:252-263. doi:10.1590/1516-3180.2014.00351712
38. CADTH. Aripiprazole (Abilify): depression, Major Depressive Disorder (MDD). Aripiprazole (Abilify): depression, Major Depressive Disorder (MDD). Ottawa (ON). 2016. Available from: https://www.ncbi.nlm.nih. gov/pubmed/28121104. Accessed October 8, 2019.

39. Yokoi Y, Sumiyoshi T. Application of transcranial direct current stimulation to psychiatric disorders: trends and perspectives. Neuropsychiatr Electrophysiol. 2015;1(1):10. doi:10.1186/s40810015-0012-x

40. D’Urso G, Dell'Osso B, Rossi R, et al. Clinical predictors of acute response to transcranial direct current stimulation (tDCS) in major depression. J Affect Disord. 2017;219:25-30. doi:10.1016/j. jad.2017.05.019

\section{Publish your work in this journal}

Neuropsychiatric Disease and Treatment is an international, peerreviewed journal of clinical therapeutics and pharmacology focusing on concise rapid reporting of clinical or pre-clinical studies on a range of neuropsychiatric and neurological disorders. This journal is indexed on PubMed Central, the 'PsycINFO' database and CAS, and is the official journal of The International Neuropsychiatric Association (INA). The manuscript management system is completely online and includes a very quick and fair peer-review system, which is all easy to use. Visit http://www.dovepress.com/testimonials.php to read real quotes from published authors. 